\title{
Learning Object Context for Dense Captioning
}

\author{
Xiangyang Li, ${ }^{1,2}$ Shuqiang Jiang, ${ }^{1,2}$ Jungong $\mathbf{H a n}^{3}$ \\ ${ }^{1}$ Key Laboratory of Intelligent Information Processing of Chinese Academy of Sciences (CAS), \\ Institute of Computing Technology, CAS, Beijing, 100190, China \\ ${ }^{2}$ University of Chinese Academy of Sciences, Beijing, 100049, China \\ ${ }^{3}$ School of Computing and Communications, Lancaster University, United Kingdom \\ xiangyang.li@vipl.ict.ac.cn, sqjiang@ ict.ac.cn, jungonghan77@gmail.com
}

\begin{abstract}
Dense captioning is a challenging task which not only detects visual elements in images but also generates natural language sentences to describe them. Previous approaches do not leverage object information in images for this task. However, objects provide valuable cues to help predict the locations of caption regions as caption regions often highly overlap with objects (i.e. caption regions are usually parts of objects or combinations of them). Meanwhile, objects also provide important information for describing a target caption region as the corresponding description not only depicts its properties, but also involves its interactions with objects in the image. In this work, we propose a novel scheme with an object context encoding Long Short-Term Memory (LSTM) network to automatically learn complementary object context for each caption region, transferring knowledge from objects to caption regions. All contextual objects are arranged as a sequence and progressively fed into the context encoding module to obtain context features. Then both the learned object context features and region features are used to predict the bounding box offsets and generate the descriptions. The context learning procedure is in conjunction with the optimization of both location prediction and caption generation, thus enabling the object context encoding LSTM to capture and aggregate useful object context. Experiments on benchmark datasets demonstrate the superiority of our proposed approach over the state-of-the-art methods.
\end{abstract}

\section{Introduction}

Over the past few years, significant progress has been made in image understanding. In addition to image classification (Krizhevsky, Sutskever, and Hinton 2012; Simonyan and Zisserman 2015; Hu, Shen, and Sun 2018), object detection (Ren et al. 2015; Redmon et al. 2016; Hu et al. 2018) and image captioning (Karpathy and Fei-Fei 2015; Xu et al. 2015; Gu et al. 2018), some more challenging tasks such as scene graph generation (Xu et al. 2017; Li et al. 2017; Klawonn and Heim 2018) and visual question answering (Shih, Singh, and Hoiem 2016; Anderson et al. 2018) can be implemented by state-of-the-art visual understanding systems. In order to reveal more details in images and alleviate the problem of reporting bias (Gordon and Van Durme 2013), dense captioning (Johnson, Karpathy, and Fei-Fei 2016;

Copyright (C) 2019, Association for the Advancement of Artificial Intelligence (www.aaai.org). All rights reserved.
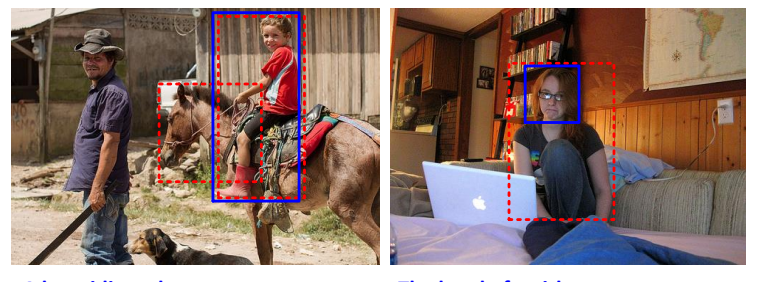

A boy riding a horse. (person, horse)

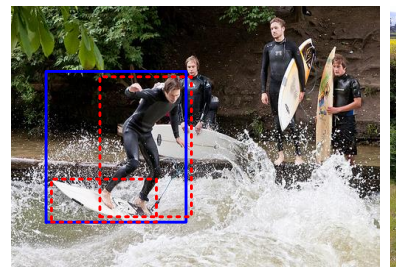

Surfer with part of board out of water. (person, surferboard)

Caption Region (person)

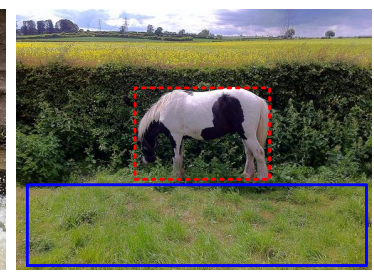

Grass near the horse.

(horse)

:..................... Contextual Object
The head of a girl.

Figure 1: Illustration of caption regions and contextual objects which provide valuable cues for predicting locations and generating the target descriptions (Best viewed in color).

Yang et al. 2017) aims to describe the content of images at local region level, which not only detects visual elements but also generates natural language sentences to describe them.

Dense captioning is similar to object detection in the sense that they both need to locate regions of interest in images. The difference is that dense captioning generates natural language descriptions for regions while object detection assigns fixed class labels to them. Currently, the prevailing framework for dense captioning consists of three components: (i) A region proposal component is used to reduce the number of candidates to be described, which is similar to the region proposal network (RPN) (Ren et al. 2015) in object detection. (ii) A language component is used to generate descriptions for all the proposals. (iii) A regression component is used to predict the bounding boxes of the captioned region candidates. Johnson et al. (Johnson, Karpathy, and FeiFei 2016) utilize a fully convolutional localization network for this task. As bounding boxes of target regions are highly overlapped with each other and the annotated concepts are 

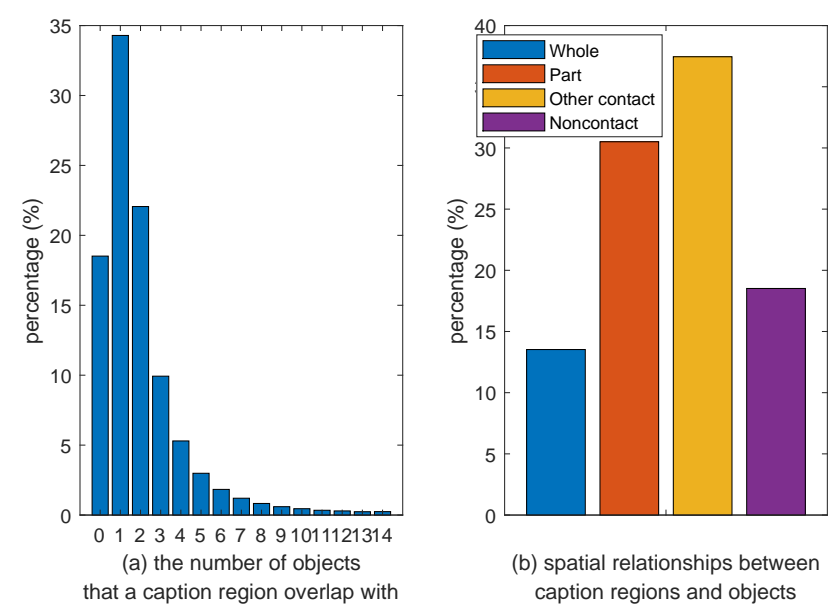

Figure 2: The spatial distributions of caption regions and objects in ground truth annotations on the VG-COCO dataset.

huge, Yang et al. (Yang et al. 2017) add a joint inference module where localizing bounding boxes is jointly predicted from the features of the target regions and features of the predicted descriptions. In their work, the whole image is used as context to help locate and describe caption regions.

Previous work either uses the local appearance independently or just complements it with the whole image as the context to locate and describe caption regions. In this paper, we propose a framework to exploit object context for dense captioning, transferring the knowledge from objects to caption regions. Regions in dense captioning have correlations with objects from object detection mainly in two aspects.

The first one is that caption regions and objects have high overlaps in spatial locations. For example, as shown in the right of the first row in Figure 1, the region with the description of "the head of a girl" is a part of the object instance of "person", which is completely contained by the object. Some caption regions are almost the same with objects in locations or are the combinations of several objects. To get a holistic view, we present the statistical information of overlaps between caption regions and objects in the VG-COCO dataset (which will be elaborated in our experiments). Figure 2 (a) illustrates the number of objects that a caption region overlap with. It can be observed that most of the caption regions $(86.5 \%)$ overlap with objects in spatial locations with the number of objects varying from 1 to 5 . We categorize these caption regions according to their spatial relationships with objects into four types: (i) Caption regions which are almost the whole objects (Whole): They have Intersectionover-Union (IoU) values with objects that are bigger than 0.7 , as this value is widely used to evaluate the effectiveness of a detected region in object detection (Girshick et al. 2014; Ren et al. 2015). (ii) Caption regions which are parts of objects (Part): They are completely contained in objects and the IoU values between them are smaller than 0.7. (iii) Caption regions which have spatial contact with objects but they are neither the whole objects nor parts of objects (Other contact). (iv) Caption regions have no spatial contact with objects (Noncontact): They are usually abstract concept regions which have no overlap with any objects (i.e. sky, grass and street). The examples in Figure 1 correspond to these four kinds of relationships. Figure 2 (b) shows the percentages of these four kinds of regions. Objects in an image provide useful indications to predict caption regions. For example, caption regions which are parts of objects appear in very distinctive locations within objects. As shown in the right of the first row of Figure 1, the caption region of a head usually tends to be near the upper middle of the person object.

The second aspect is that the descriptions for caption regions and objects have commonalities in semantic concepts. The descriptions for caption regions not only describe the properties such as color, shape and texture, but also involve their interactions with the surrounding objects. For example, in the first row of Figure 1, the description for the boy also involves its relationship (i.e. riding) with the horse. Even for the abstract concepts regions (i.e. Noncontact), their predictions are closely related with the objects in the image.

Hence, objects provide valuable cues to help locate caption regions and generate descriptions for them. To capture useful object information in an image, we propose a novel framework to learn complementary object context for each caption region, as shown in Figure 3. We first detect a set of objects, and then all the objects are put into a context encoding Long Short-Term Memory network to form informative context. The LSTM cell progressively takes each object as inputs and decides whether to retain the information from the current input or discard it, based on the information captured from its previous states and current input. At last, the learned context is used as guided information to help generate the descriptions and predict the bounding box offsets. The results show that the learned context is beneficial to describe and locate caption regions.

In summary, our contributions are as follows: First, we propose an architecture to model complementary object context for dense captioning. As dense captioning and object detection are image understanding tasks at different levels, experiments demonstrate that bringing features from object detection can benefit dense captioning. Second, we explore different architectures to fuse the learned context features and the features of caption regions and analyze the mechanisms for them. We also visualize the internal states of the context encoding LSTM cells. The visualization shows that our model can automatically select the relevant context objects to form discriminative and informative context features.

\section{Related work}

Image Captioning. Many approaches have been explored for the task of image captioning, which aims to generate natural language descriptions for the whole image. Most prevailing methods are based on the CNN-RNN framework. Karpathy et al. (Karpathy and Fei-Fei 2015) use a deep CNN to extract visual features and put these features into an RNN as the initial start word to generate image descriptions. Jin et al. (Fu et al. 2017) utilize the attention mechanism to change gaze on different objects when generating 


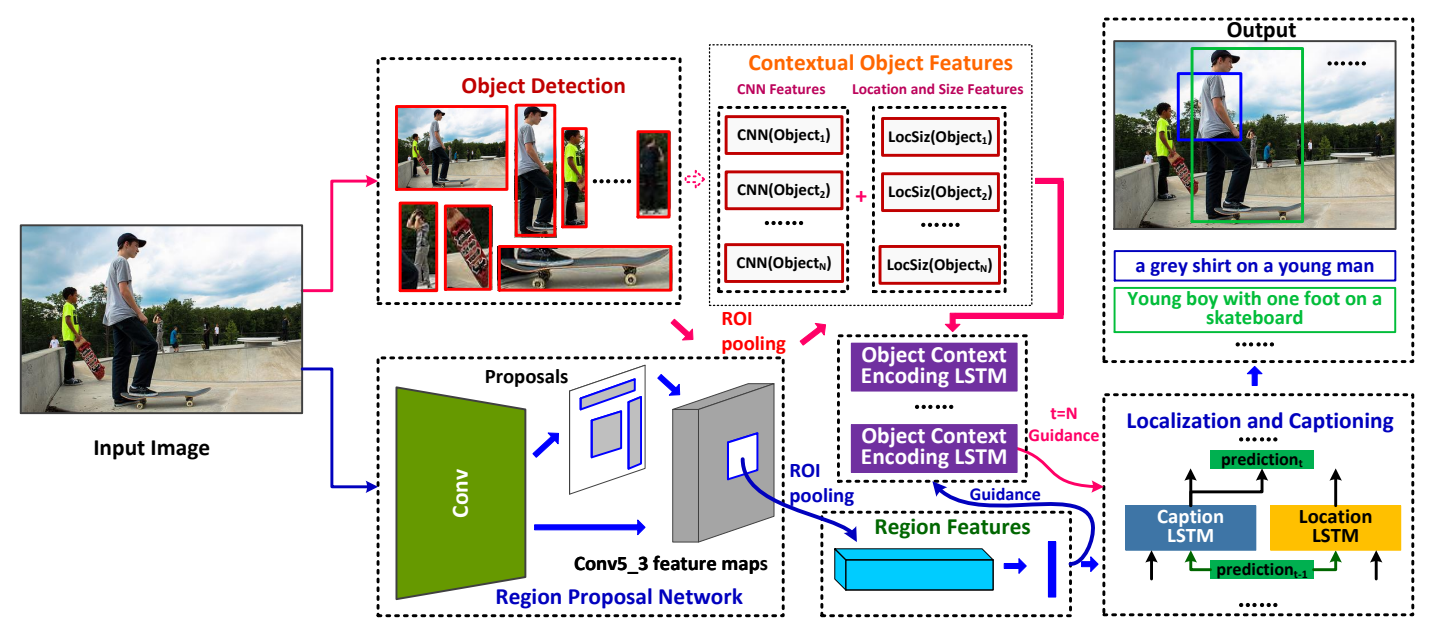

Figure 3: Overview of our framework. A region proposal network (RPN) is used to generate regions of interest (ROIs). To leverage objects information in the image, a pre-trained Faster R-CNN model is used to detect objects in the image. For both caption regions and detected objects, ROI pooling is used on top of the shared last convolutional layer (i.e. conv5_3) to get CNN features. The combination of CNN features and location and size features is used to represent each object. The features of all objects are arranged as a sequence and are put into the object context encoding LSTM with the guidance of the region features to form the complementary object context features. At last, the features of the target regions and the learned context are used to generate descriptions and bounding boxes.

each word in the sentence. Gan et al. (Gan et al. 2017) generate sentences based on detected high-level semantic concepts. In order to optimize captioning models on test metrics (e.g. CIDEr, SPICE), many methods based on Reinforcement Learning (RL) have been proposed (Rennie et al. 2017; Luo et al. 2018). In this paper, we address the task of dense captioning. For each caption region, we use the features of target regions as guidance to learn the corresponding complementary object context. After getting the learned context, we then use the context to guide the prediction of its description and bounding box offset.

Object Detection. The task of object detection identifies a set of objects in the given image, providing both their predefined categories and the corresponding bounding box offsets. There has been a lot of significant progress in object detection (Redmon et al. 2016; Liu et al. 2016; Hu et al. 2018). The first attractive work is the combination of region proposals and CNNs (R-CNN) (Girshick et al. 2014). To achieve the goal of processing regions in an image only with a single feedforward pass of the CNN, Girshick et al. (Girshick 2015) have modified this pipeline by sharing computation of convolutionals. Some further approaches explore region proposal strategies based on neural networks without extra algorithms to hypothesize object locations (Ren et al. 2015; Redmon et al. 2016). Our work is built on top of the region proposal network (RPN) of Faster R-CNN (Ren et al. 2015). Similar to our work, Chen et al. (Chen and Gputa 2017) propose a Spatial Memory Network (SMN) to model objectlevel context to improve modern object detectors. In this work, we automatically learn complementary object context to help predict more precise locations and generate better descriptions for dense captioning.

\section{Our Approach}

The overall architecture is shown in Figure 3. In this section, we will describe each component of the proposed approach.

\section{Region Proposal Network}

We use VGG-16 (Simonyan and Zisserman 2015) as the base network. For the region proposal network, we apply the method proposed by Ren et al. (Ren et al. 2015) to generate regions of interest for dense captioning. It has 3 convolutional layers where the first one transforms the features of conv5_3 to suitable representations with filter size $3 \times 3$. And the remaining two layers with filter size $1 \times 1$ are for foreground/background classification and bounding box regression respectively. At each location, anchors with different scales and ratios are used to generate possible regions.

During training and testing, the region proposal network outputs a number of rectangular region proposals. We feed each proposal to the ROI pooling layer to obtain its feature cube. Afterwards, they are flattened into a vector and pas608sed through 3 fully-connected layers. In this way, for each generated caption region $i$, we can obtain a vector $V_{i}$ of dimension $D=512$ that compactly encodes its visual appearance.

\section{Object Context Encoding}

As objects in images not only provide valuable cues for locating caption regions but only offer useful information for describing them, it is important to model the context among objects for dense captioning. In order to model different object context for each caption region, we use the LSTM which updates with guidance information (i.e. gLSTM) (Jia et al. 2015) as our object context encoding module. It takes the 


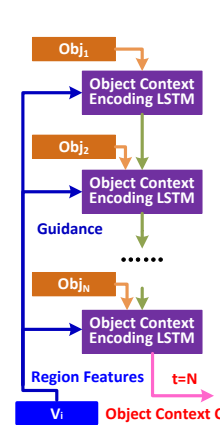

(a)

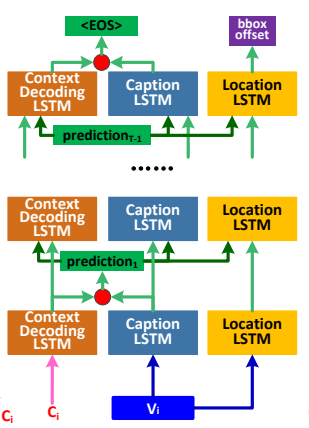

(b)

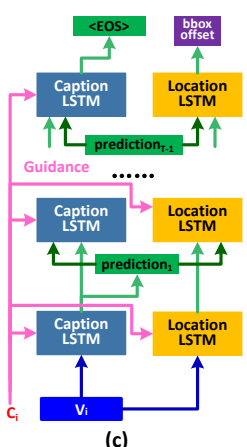

(c)
Figure 4: Illustration of object context encoding network module and region caption generation and localization module: (a) Object context encoding, (b) Object context decoded with a LSTM, and (c) Object context used as guidance.

output of the previous time step, the input of the current time step and the fixed guidance as the its current inputs. The update equations at time $t$ can be formulated as:

$$
\begin{aligned}
i_{t} & =\sigma\left(\mathbf{W}_{i x} x_{t}+\mathbf{W}_{i m} m_{t-1}+\mathbf{W}_{i g} \mathbf{g}\right) \\
f_{t} & =\sigma\left(\mathbf{W}_{f x} x_{t}+\mathbf{W}_{f m} m_{t-1}+\mathbf{W}_{f g} \mathbf{g}\right) \\
o_{t} & =\sigma\left(\mathbf{W}_{o x} x_{t}+\mathbf{W}_{o m} m_{t-1}+\mathbf{W}_{o g} \mathbf{g}\right) \\
c_{t} & \left.=f_{t} \odot c_{t-1}+i_{t} \odot \phi\left(\mathbf{W}_{c x} x_{t}+\mathbf{W}_{c m}+\mathbf{W}_{c g} \mathbf{g}\right)\right) \\
m_{t} & =o_{t} \odot \phi\left(c_{t}\right)
\end{aligned}
$$

where $g$ denotes the guidance information, $t$ ranges from the start of the input sequence to the end of it; $i_{t}, f_{t}$ and $o_{t}$ represent the input gate, forget gate, and output gate at time step $t ; c_{t}$ is the memory cell and $m_{t}$ is the hidden state; $\odot$ represents the element-wise multiplication, $\sigma(\cdot)$ represents the sigmoid function and $\phi(\cdot)$ represents the hyperbolic tangent function; $W_{[\cdot][\cdot]}$ denote the parameters of the model. In this work, we use CNN features of the target region as the guidance for the object context encoding LSTM.

In order to obtain rich object information, we use a pretrained Faster R-CNN to extract a set of objects from the image. Like previous approaches (Fu et al. 2017), the entire image is also utilized as a specific object region. We then put its bounding box offset of each object $j$ to the ROI pooling layer and the 3 fully-connected layers (i.e. the same architecture used to extract the feature vectors for caption regions) to obtain the features $v o_{j}$ from the shared conv5_3 layer. To acquire more information, we also extract location and size features $l_{j}$ for each object $j$ :

$$
l_{j}=\left[\frac{x_{t l}}{W}, \frac{y_{t l}}{H}, \frac{x_{b r}}{W}, \frac{y_{b r}}{H}, \frac{j_{j} \cdot h_{j}}{W \cdot H}\right]
$$

where $x$ and $y$ are the locations of the top left and bottom right corners of the object $j, w_{j}$ and $h_{j}$ are the width and height of $j, W$ and $H$ are the width and height of the image. We then concatenate the CNN features $v o_{j}$, the location and size features $l_{j}$ to represent object $j$. At last, we use a fullyconnected layer to transform the concatenated features to the final representations $o b j_{j}$ which has the same size with $V_{i}$.

$$
o b j_{j}=\mathbf{W}_{o b}\left[v o_{j}, l_{j}\right]+\mathbf{b}
$$

where [.] denotes the concatenation operation, $\mathbf{W}_{o b}$ and $\mathbf{b}$ are the parameters for the fully-connected layer.

After we get the features for each detected object, we arrange them as a sequence which will be fed into the object context encoding LSTM. As the spatial information of objects is an important information for many visual tasks (Bell et al. 2016; Chen and Gputa 2017), we sort these objects by their locations in the image. More accurately, they are arranged with the order of from left to right and top to down. Some other orders (e.g. area order, confidence order) will also be explored in our experiments. In this way, given an image $I$, we utilize $\operatorname{seq}(I)$ to denote its object information, which contains a sequence of representations $\operatorname{seq}(I)=\left\{o b j_{1}, o b j_{2}, \ldots, o b j_{N}\right)$. For the object context decoding procedure, we use the features of the target caption region $v_{i}$ as the guidance at each step, as illustrated in Figure 4 (a). The object context encoding LSTM takes in seq $(I)$ progressively and encodes each object into a fixed length vector. Thus, we can obtain the encoding hidden states computed from:

$$
\begin{aligned}
h_{e n_{t}}= & \operatorname{LSTM}_{e n}\left(\operatorname{seq}(I)_{t}, V_{i}, h_{e n_{t-1}}\right), \\
& t=1,2, \ldots N
\end{aligned}
$$

where $N$ is the number of objects. Once the hidden representations from all the context objects are obtained, we use the last the hidden state to represent the complementary object context $c_{i}$ for the caption region $i: C_{i}=h_{e n_{N}}$. The features for the caption region $V_{i}$ and the corresponding context $C_{i}$ will be used in the next stage to generate the description and predict the bounding box offset.

\section{Region Caption Generation and Localization}

The description for a caption region usually depicts its properties and its interactions with objects in the image. So object context is of great importance for describing a caption region. It is an essential part of the input information which provides a broader understanding of the image and delivers visual cues to generate better descriptions.

We explore two different architectures to leverage the features of caption region $V_{i}$ and the learned object context $C_{i}$ to generate an appropriate description for each caption region $i$. The first architecture uses a LSTM to decode the object context into recurrent representations, of which the outputs are fused with the outputs of the caption LSTM. At each time step, the concatenation of them is utilized to generate the description, as shown in Figure 4 (b). As the learned complementary object context is decoded with a LSTM, this architecture is denoted as COCD for convenience. This one is similar to the architecture used in (Yang et al. 2017), where they use the whole image as the context but our context is automatically learned from objects in the image. In the second architecture, the context is used as guidance information which is fed into the caption LSTM, as shown in Figure 4 (c). It is denoted as COCG for convenience. For the region caption generation procedure, the learned complementary object context works at a global level and guides the caption LSTM at each time step to encode the features of the region into a language description. The motivation for 
COCG is as follows: Because COCD has four LSTMs, it is hard to train. Removing the context decoding LSTM will alleviate the vanishing gradient problem.

For region localization, our networks are designed with the same spirits in (Yang et al. 2017). The features of the region $V_{i}$ are put into the location LSTM as an initial word. In the following steps, it progressively receives the outputs of the caption LSTM. After all of the predicted words (except the token $\langle E O S\rangle$ which indicates the end of a sentence) are put into the location LSTM, the last hidden state is used to predict the box offset. In this manner, the predicted box offset is interrelated with the corresponding description.

\section{Training and Optimization}

To obtain objects in images, we train a Faster CNN (Ren et al. 2015) model using the ResNet-101 (He et al. 2016). The model is first pre-trained on ImageNet (Russakovsky et al. 2015) dataset and then is fine-tuned on MS COCO (Lin et al. 2014) (except the test and validation images in VG-COCO). For each image, we detect 10 objects with high confidence. Because each image has an average of 7 objects (which will be introduced our experiments), most of objects will be covered. For the RPN, 12 anchors are used to generate possible proposals at each location and 256 boxes are sampled for each forward procedure. The size of our vocabulary is 1000 . The max length of all the sentence is set to 10 . For all of the LSTM networks, the size of the hidden state is set to 512 . We train the full model end-to-end in a single step of optimization. Our training batches consist of a single image that has been resized so that the longer side has 720 pixels.

\section{Experiments}

\section{Datasets and Evaluation Metrics}

We use the Visual Genome (VG) dataset (Krishna et al. 2017) and the VG-COCO dataset which is the intersection of VG V1.2 and MS COCO (Lin et al. 2014) as the evaluation benchmarks.

VG. Visual Genome currently has three versions: V1.0, $\mathrm{V} 1.2$ and V1.4. As the region descriptions in V1.4 are the same with the region descriptions in V1.2, we conduct our experiments on V1.0 and V1.2. The training, validation and test splits are the same with (Johnson, Karpathy, and FeiFei 2016). There are 77,398 images for training and 5,000 images for validation and test respectively.

VG-COCO. Even though Visual Genome provides object annotations for each image, the target bounding boxes are much denser than the bounding boxes in other object detection benchmark datasets such as MS COCO (Lin et al. 2014) and ImageNet (Russakovsky et al. 2015). For example, each image in the training set of VG V1.2 contains an average of 35.4 objects, but the average value for MS COCO is only 7.1. To get proper object bounding boxes and caption region bounding boxes for each image, the intersection of VG V1.2 and MS COCO is used in our paper, which is denoted as VGCOCO. There are 38,080 images for training, 2,489 images for validation and 2,476 for test.

For evaluation, we use mean Average Precision (mAP), which is same with (Johnson, Karpathy, and Fei-Fei 2016;

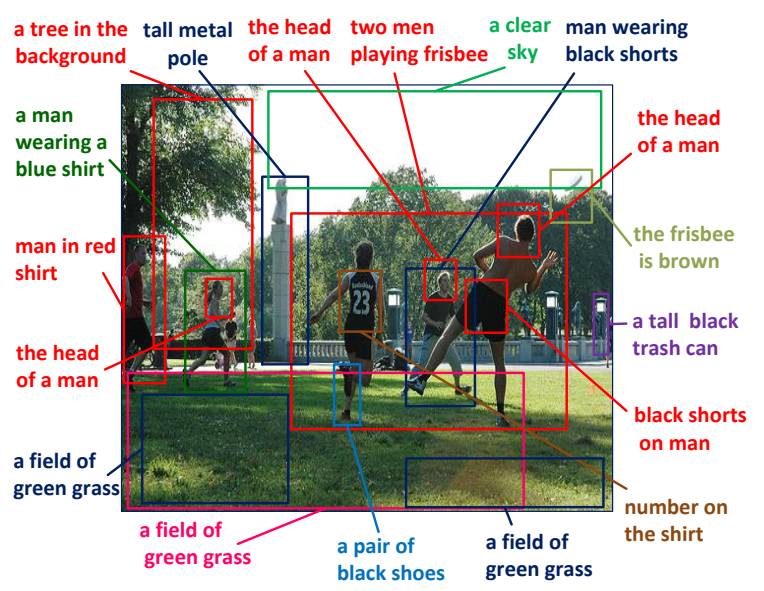

Figure 5: Illustration of detected regions and the corresponding descriptions generated by our method. Predictions with high confidence scores are represented.

Table 1: The results on the VG-COCO dataset (\%).

\begin{tabular}{|c|c|}
\hline Method & mAP \\
\hline FCLN (Johnson, Karpathy, and Fei-Fei 2016) & 4.23 \\
\hline JIVC (Yang et al. 2017) & 7.85 \\
\hline Max pooling & 7.86 \\
\hline COCD & 7.92 \\
\hline COCG & $\mathbf{8 . 9 0}$ \\
\hline ImgG & 7.81 \\
\hline \hline COCG-LocSiz & 8.76 \\
\hline COCG\&GT & 9.79 \\
\hline
\end{tabular}

Yang et al. 2017). It measures localization and description accuracy jointly. Average precision is computed for different IoU thresholds and different Meteor scores (Lavie and Agarwal 2007), then averaged to produce the mAP score. For localization, IoU thresholds $0.3,0.4,0.5,0.6,0.7$ are used. Meanwhile, Meteor scores 0, 0.05, 0.10, 0.15, 0.20, 0.25 are used for language similarity. For each test image, top 300 boxes with high confidence after non-maximum suppression (NMS) with IoU of 0.7 are generated. The beam search size is set to 1 when generating descriptions. The final results are generated with a second round of NMS with IoU of 0.3.

\section{Effectiveness of the Learned Object Context}

In this section, we evaluate the effectiveness of the complementary object context learned by our methods on the VGCOCO dataset. The results are shown in Table 1. We use the method proposed by Yang et al. (Yang et al. 2017) as our baseline method which incorporates joint inference and visual context fusion (denoted as JIVC). The performance of fully convolutional localization network (denoted as FCLN) proposed by Johnson et al. (Johnson, Karpathy, and Fei-Fei 2016) is also provided. The mAP scores for different variants of models are shown in Table 1.

As shown in Table 1, the learned complementary object context helps to improve mAP scores with gains ranging 
from 0.07 to 1.05 . Several conclusions can be obtained: (i) The learned object context is effective, demonstrating its complementarity to the caption region features and its superiority to the whole image context. For the architecture where the learned context is decoded with a LSTM (COCD), it surpasses the baseline with an improvement of 0.07. It demonstrates that the learned context is better than using the whole image as the context. While the method using max pooling to obtain the context (Max pooling) almost gets the same performance with the baseline, this demonstrates that it is better to use the proposed method to model the context. (ii) The architecture which uses the learned context as guidance (COCG) gets the best performance. COCG injects the context into the caption LSTM and location LSTM as guidance. Meanwhile, the context learning procedure is bundled together with location prediction and caption generation. In this way, the context encoding LSTM is adapted to the caption and location LSTMs, and thus captures and aggregates useful context. (iii) The improvements of COCG mainly come from the learned region specific context. The architecture which uses the whole image as contextual information for COCG (ImgG) almost has the same performance with JIVC. Because the whole image context is fixed for each caption region, using the whole image context as the guidance is not superior to decoding it with an extra LSTM (as JIVC is a litter better than ImgG). The results demonstrate that the improvements of COCG mainly come from the learned region specific context instead of the utilization of gLSTM. (iv) The results show that only using the CNN features to represent objects (COCG-LocSiz) leads a little drop in the performance. The experiments also show that learning the complementary object context on the ground truth objects (COCG\&GT) can obtain better performance. It demonstrates that accurate object bounding boxes are beneficial to obtain better results.

Figure 5 shows some examples generated by our method which automatically learns complementary object context for each caption region. Figure 6 shows quantitative comparison between the baseline (JIVC) and our method (COCG). With the Meteor score of 0 , our proposed method obtains promising improvements. The results show that our method can obtain better locations. Meanwhile, it is illustrated that the proposed method obtains better performance at all settings, demonstrating that our complementary object context is useful for both describing and locating caption regions. Figure 7 shows several examples which qualitatively demonstrate that our method is better both at generating descriptions and predicting bounding box offsets. The last column in Figure 7 shows a negative example, as the generated caption of COCG is slightly worse than the one of JIVC.

\section{Context Encoding with Different Orders}

For the evaluations above, we only use the location order to arrange the detected objects. In this section, we evaluate the performance when the objects are sorted with different orders. Besides location order which has been used, we explore the other two kinds of orders: area and confidence. Area order is that context objects are sorted by their areas from big to small, as we often focus on big objects

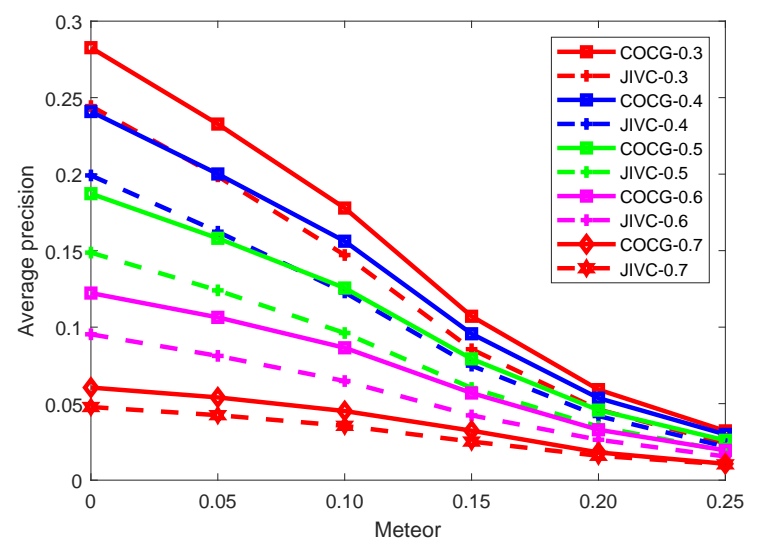

Figure 6: Average precision with different Meteor scores and different IoU thresholds on the VG-COCO dataset.

Table 2: The mAP performance on the VG-COCO dataset when context objects are arranged with different orders (\%).

\begin{tabular}{|c|c|}
\hline Method & mAP \\
\hline Location & $\mathbf{8 . 9 0}$ \\
\hline Area & 8.74 \\
\hline Confidence & 8.89 \\
\hline
\end{tabular}

and neglect small objects. Confidence order is that context objects are sorted with their confidence scores from big to small, where the confidence scores are provided by the object detection model. Table 2 shows the mAP for different orders. It can be observed that location order achieves the best performance and the other two also obtain comparable performance. So in the following sections, we only use the location order to arrange the objects.

\section{Results on Visual Genome}

We also evaluate the effectiveness of our method on Visual Genome (VG) dataset. The results are shown in Table 3. Leveraging the object context in the image, we achieve stateof-the-art performance. The proposed method obtains a relative gain of $5.5 \%$ on VG V1.0 and a relative gain of $4.3 \%$ on VG V1.2. The gains are smaller than the gain in VG-COCO. The reasons are follows: First, the object detection model is only trained on MS COCO thus it have no knowledge about objects information in VG. Second, for each image, we only detect 9 images. We have not experimented with the number of objects, it is possible that the improved recall from additional objects may improve the performance.

\section{Visualized Results}

As the gates of the LSTM architecture (whose activations range from 0 to 1) store read, write and reset information for its memory cells, the power multiplicative interactions enable the LSTM architecture to capture rich contextual information from its input sequence (Palangi et al. 2016). In this section, we examine the temporal evolution of internal gate states (i.e. input gate) and qualitatively reveal how the 

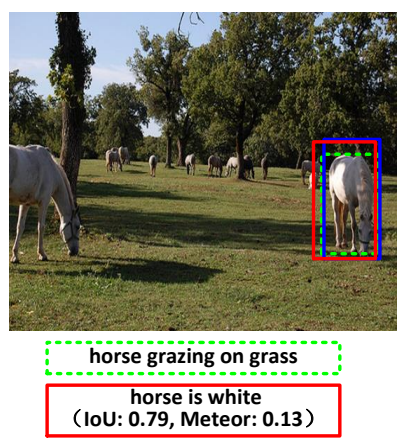

horse is eating grass IoU: 0.80, Meteor: 0.24)
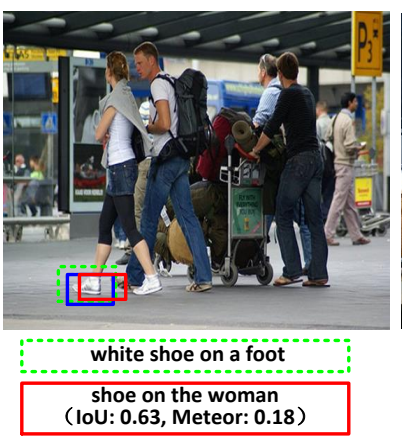

white shoe on the women white shoe on the women
(loU: 0.68 , Meteor: 0.33 )

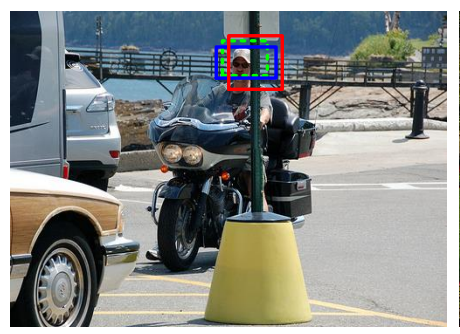

a baseball hat the guy is wearing

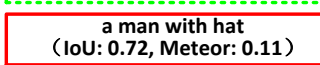

(loU: 0.72, Meteor: 0.11$)$ man is wearing a white hat
(IoU: 0.75, Meteor: 0.19 )

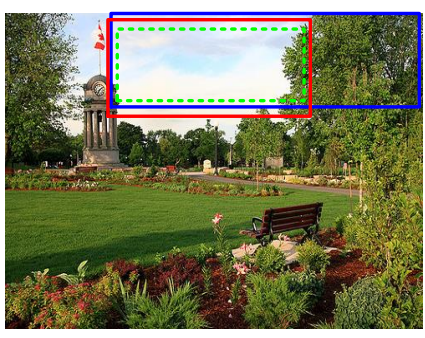

thin clouds in the sky

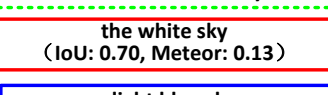
a light blue sky
(lou: 0.50,

Figure 7: Qualitative comparisons between baseline (JIVC) and our method (COCG). The green box, the red box and the blue box are the grounding truth, the prediction of JIVC and the prediction of COCG respectively (Best viewed in color).

Table 3: The mAP performance on V1.0 and V $1.2(\%){ }^{*}$ indicates that the results are obtained by our implementation.

\begin{tabular}{|c|c|c|}
\hline Method & VG V1.0 & VG V1.2 \\
\hline FCLN (Johnson et al. 2016) & 5.39 & - \\
\hline FCLN $^{*}$ (Johnson et al. 2016) & 5.02 & 5.16 \\
\hline JIVC (Yang et al. 2017) $^{\text {(Y) }}$ & 9.31 & 9.96 \\
\hline JIVC $^{*}$ (Yang et al. 2017) & 9.28 & 9.73 \\
\hline ImgG & 9.25 & 9.68 \\
\hline COCD & 9.36 & 9.75 \\
\hline COCG & $\mathbf{9 . 8 2}$ & $\mathbf{1 0 . 3 9}$ \\
\hline
\end{tabular}

object context encoding LSTM retains valuable context information and attenuates unimportant information. Figure 8 shows two examples from the test set of VG-COCO. For the example in the first row of Figure 8, to obtain the complementary object context for the region which is described as "a man wearing shorts", the LSTM selects and propagates the most relevant object which is labeled as 5 (an object instance of person), as the mean input gate activations for this object is bigger than others. While for the example in the second row, the LSTM has big input gate activations for the object which is labeled as 6 . The visualization indicates that meaningful patterns can be learned by the context encoding LSTM. By automatically selecting and attenuating context objects, the context encoding LSTM can generate rich complementary object context for each caption region.

\section{Conclusion}

In this work, we have demonstrated the importance of learning object context for dense captioning, as caption regions and objects not only have a high degree of overlap in their spatial locations but also have a lot of commonalities in their semantic concepts. To transfer knowledge from detected objects to caption regions, we introduce a framework with an object context encoding LSTM module to explicitly learn complementary object context for locating and describing each caption region. The object context encoding LSTM progressively receives features from context objects to cap-

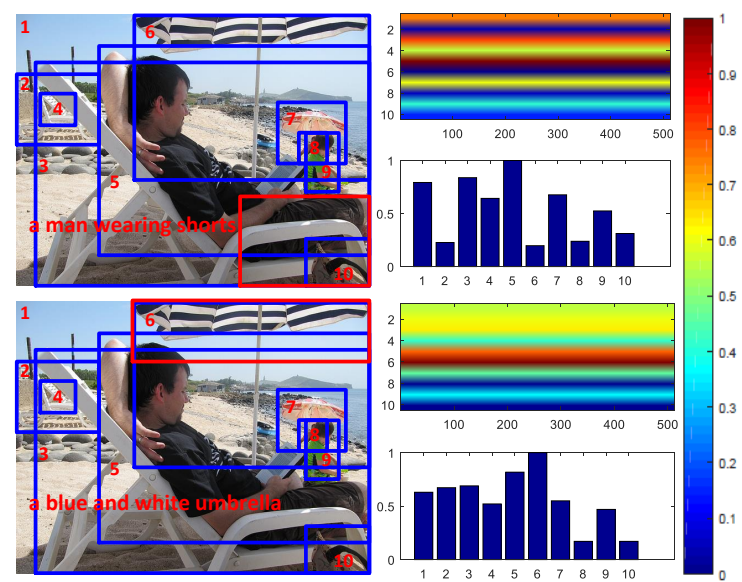

Figure 8: The visualization of the object context encoding LSTM. The first column shows the images, where blue boxes are the detected objects, the red box is the target caption region and the text is the generated description. The top of the second column shows the mean input gate activations for each object and the bottom shows the min-max normalization of them. Because the hidden state size of the object context encoding LSTM is 512, the gate activations are 512 dimensional vectors (Best viewed in color).

ture and aggregate rich object context features. After getting the learned complementary object context features, the context features and the region features are effectively combined to predict descriptions and locations. We explore the capabilities of different architectures and achieve promising results on benchmark datasets. In future work, we will exploit high-level semantic information from objects to obtain more useful cues for locating and describing caption regions.

\section{Acknowledgements}

This work was supported in part by the National Natural Science Foundation of China under Grant 61532018, in part by the Lenovo Outstanding Young Scientists Program, in part 
by National Program for Special Support of Eminent Professionals and National Program for Support of Top-notch Young Professionals.

\section{References}

Anderson, P.; He, X.; Buehler, C.; Teney, D.; Johnson, M.; Gould, S.; and Zhang, L. 2018. Bottom-up and top-down attention for image captioning and visual question answering. In CVPR, 6077-6086.

Bell, S.; Zitnick, C. L.; Bala, K.; and Girshick, R. 2016. Inside-Outside Net: Detecting objects in context with skip pooling and recurrent neural networks. In CVPR, 28742883.

Chen, X., and Gputa, A. 2017. Spatial memory for context reasoning in object detection. In ICCV, 4106-4116.

Fu, K.; Jin, J.; Cui, R.; Sha, F.; and Zhang, C. 2017. Aligning where to see and what to tell: image captioning with region-based attention and scene-specific contexts. IEEE Transactions on Pattern Analysis and Machine Intelligence 39(12):2321-2334.

Gan, Z.; Gan, C.; He, X.; Pu, Y.; Tran, K.; Gao, J.; Carin, L.; and Deng, L. 2017. Semantic compositional networks for visual captioning. In CVPR, 2630-2639.

Girshick, R.; Donahue, J.; Darrell, T.; and Malik, J. 2014. Rich feature hierarchies for accurate object detection and segmentation. In CVPR, 580-587.

Girshick, R. 2015. Fast R-CNN. In ICCV, 1440-1448.

Gordon, J., and Van Durme, B. 2013. Reporting bias and knowledge acquisition. In Automated knowledge base construction Workshop, 25-30.

Gu, J.; Cai, J.; Wang, G.; and Chen, T. 2018. Stackcaptioning: Coarse-to-fine learning for image captioning. In AAAI, 6837-6844.

He, K.; Zhang, X.; Ren, S.; and Sun, J. 2016. Deep residual learning for image recognition. In $C V P R, 770-778$.

Hu, H.; Gu, J.; Zhang, Z.; Dai, J.; and Wei, Y. 2018. Relation networks for object detection. In CVPR, 3588-3597.

Hu, J.; Shen, L.; and Sun, G. 2018. Squeeze-and-excitation networks. In $C V P R, 7132-7141$.

Jia, X.; Gavves, E.; Fernando, B.; and Tuytelaars, T. 2015. Guiding Long-Short Term Memory for image caption genaration. In $I C C V, 2407-2415$.

Johnson, J.; Karpathy, A.; and Fei-Fei, L. 2016. Densecap: Fully convolutional localization networks for dense captioning. In CVPR, 4565-4574.

Karpathy, A., and Fei-Fei, L. 2015. Deep visual-semantic alignments for generating image descriptions. In $C V P R$, 3128-3137.

Klawonn, M., and Heim, E. 2018. Generating triples with adversarial networks for scene graph construction. In $A A A I$, 6992-6999.

Krishna, R.; Zhu, Y.; Groth, O.; Johnson, J.; Hata, K.; Kravitz, J.; Chen, S.; Kalantidis, Y.; Li, L.-J.; Shamma, D. A.; Bernstein, M.; and Fei-Fei, L. 2017. Visual Genome: Connecting language and vision using crowdsourced dense image annotations. International Journal of Computer Vision 123(1):32-73.

Krizhevsky, A.; Sutskever, I.; and Hinton, G. E. 2012. Imagenet classification with deep convolutional neural networks. In NIPS, 1097-1105.

Lavie, A., and Agarwal, A. 2007. METEOR: An automatic metric for MT evaluation with high levels of correlation with human judgments. In ACL, 228-231.

Li, Y.; Ouyang, W.; Zhou, B.; Wang, K.; and Wang, X. 2017. Scene graph generation from objects, phrases and region captions. In ICCV, 1270-1279.

Lin, T.-Y.; Maire, M.; Belongie, S.; Bourdev, L.; Girshick, R.; Hays, J.; Perona, P.; Ramanan, D.; Zitnick, C. L.; and Dollár, P. 2014. Microsoft COCO: Common object in context. In ECCV, 740-755.

Liu, W.; Anguelov, D.; Erhan, D.; Szegedy, C.; Reed, S.; Fu, C.-Y.; and Berg, A. C. 2016. SSD: Single shot multibox detector. In $E C C V, 21-37$.

Luo, R.; Price, B.; Cohen, S.; and Shakhnarovich, G. 2018. Discriminability objective for training descriptive captions. In CVPR, 6964-6974.

Palangi, H.; Deng, L.; Shen, Y.; Gao, J.; He, X.; Chen, J.; Song, X.; and Ward, R. 2016. Deep sentence embedding using long short-term memory networks: Analysis and application to information retrieval. IEEE/ACM Trans. Audio, Speech, Languag. Proces. 24(4):694 - 707.

Redmon, J.; Divvala, S.; Girshick, R.; and Farhadi, A. 2016. You only look once: Unified, real-time object detection. In CVPR, 779-788.

Ren, S.; He, K.; Girshick, R.; and Sun, J. 2015. Faster RCNN: Towards real-time object detection with region proposal networks. In NIPS, 91-99.

Rennie, S. J.; Marcheret, E.; Mroueh, Y.; Ross, J.; and Goel, V. 2017. Self-critical sequence training for image captioning. In CVPR, 1179-1195.

Russakovsky, O.; Deng, J.; Su, H.; Krause, J.; Satheesh, S.; Ma, S.; Huang, Z.; Karpathy, A.; Khosla, A.; Bernstein, M.; Berg, A. C.; and Fei-Fei, L. 2015. Imagenet large scale visual recognition challenge. International Journal of Computer Vision 115(3):211 - 252.

Shih, K. J.; Singh, S.; and Hoiem, D. 2016. Where to look: Focus regions for visual question answering. In $C V P R$, 4613-4621.

Simonyan, K., and Zisserman, A. 2015. Very deep convolutional networks for large-scale image recognition. In ICLR.

Xu, K.; Ba, J.; Kiros, R.; Cho, K.; Courville, A.; Salakhutdinov, R.; Zemel, R.; and Bengio, Y. 2015. Show, attend and tell: Neural image caption generation with visual attention. In ICML, 2048-2057.

Xu, D.; Zhu, Y.; Choy, C. B.; and Fei-Fei, L. 2017. Scene graph generation by iterative message passing. In $C V P R$, 3097-3106.

Yang, L.; Tang, K.; Yang, J.; and Li, L.-J. 2017. Dense captioning with joint inference and visual context. In $C V P R$, 1978-1987. 